\title{
HYPERBOLIC, L-SPACE KNOTS AND EXCEPTIONAL DEHN SURGERIES
}

\author{
KIMIHIKO MOTEGI AND KAZUSHIGE TOHKI
}

\begin{abstract}
A knot in the 3-sphere is called an $L$-space knot if it admits a nontrivial Dehn surgery yielding an L-space. Like torus knots and Berge knots, many L-space knots admit also a Seifert fibered surgery. We give a concrete example of a hyperbolic, L-space knot which has no exceptional surgeries, in particular, no Seifert fibered surgeries.
\end{abstract}

\section{INTRODUCTION}

For any rational homology 3 -sphere $M$, the rank of the Heegaard Floer homology $\widehat{\mathrm{HF}}(M)$ is bounded below by the order of $H_{1}(M ; \mathbb{Z})$. If the rank of $\widehat{\mathrm{HF}}(M)$ is equal to the order of $H_{1}(M ; \mathbb{Z})$, then $M$ is called an $L$-space. The class of $\mathrm{L}$-spaces includes lens spaces (except $S^{2} \times S^{1}$ ), and more generally, 3-manifolds with elliptic geometry [16, Proposition 2.3]. An efficient way to find L-spaces is through "bootstrapping" a known "L-space surgeries" on a knot. A Dehn surgery is called an $L$-space surgery if the resulting 3-manifold is an L-space, and a knot admitting nontrivial L-space surgery is called an L-space knot. Since torus knots and Berge knots [3] admit surgeries yielding lens spaces, these are L-space knots. Ozsváth-Szabó [18, Proposition 9.6] ([6, Lemma 2.13]) gives us a complete information about the set of L-space surgeries on an L-space knot:

- If $K$ is a nontrivial, L-space knot of genus $g(K)$, then $r$-surgery on $K$ results in an L-space if $r \geq 2 g(K)-1$ or $r \leq-2 g(K)+1$.

This result, together with Thurston's hyperbolic Dehn surgery theorem [20, 21, 2, $19,4]$, says that each hyperbolic, L-space knot produces infinitely many hyperbolic L-spaces by Dehn surgery. For instance, a hyperbolic Berge knot produces infinitely many hyperbolic L-spaces.

Like torus knots and Berge knots, many L-space knots admit also a Seifert fibered surgery, i.e. a surgery yielding a Seifert fiber space. Among Montesinos knots, recent results of Lidman-Moore [10] and Baker-Moore [1] show that the only L-space knots are the pretzel knots $P(-2,3,2 n+1)$ and the torus knots $T_{2 n+1,2}$ $(n \geq 0)$ and their mirror images, each of which admits a Seifert fibered surgery

The author has been partially supported by JSPS Grants-in-Aid for Scientific Research (C), 26400099, The Ministry of Education, Culture, Sports, Science and Technology, Japan and Joint Research Grant of Institute of Natural Sciences at Nihon University for 2014.

2010 Mathematics Subject Classification. Primary 57M25, 57M27, 57M50 
$[16,10]$. Furthermore, in [14] one can find a large number of twist families of hyperbolic, L-space knots each of which admits also a Seifert fibered surgery. To the best of our knowledge, there is no explicitly known examples of hyperbolic, $\mathrm{L}^{-}$ space knots which have no Seifert fibered surgeries, though we expect there should be many.

Question 1.1. Does any hyperbolic, L-space knot admit also a Seifert fibered $L^{-}$ space surgery?

The aim of this note is to demonstrate:

Theorem 1.2. There exists a hyperbolic, L-space knot which has no exceptional surgeries, in particular, no Seifert fibered surgeries.

Remark 1.3. Using a cabling construction [6, Theorem 1.10], or more generally a satellite operation [8, Theorem 1.3], we can obtain a satellite (i.e. non-hyperbolic) L-space knot which has no Seifert fibered surgeries.

Acknowledgments. We would like to thank Ken Baker for useful discussion, in which we learned effective utilization of almost alternating diagrams of the unknot in a study of L-space knots suggested by Josh Greene. We gratefully acknowledge Mario Eudave-Muñoz, Katura Miyazaki and Tatsuya Tsukamoto for useful conversations. Finally we would like to thank Neil Hoffman, Kazuhiro Ichihara and Hidetoshi Masai for their help with fef.py and HIKMOT.

\section{Covering Knots And Montesinos trick}

A tangle $(B, t)$ is a pair of a 3 -ball $B$ and two disjoint arcs $t$ properly embedded in $B$. A tangle $(B, t)$ is trivial if there is a pairwise homeomorphism from $(B, t)$ to $\left(D^{2} \times I,\left\{x_{1}, x_{2}\right\} \times I\right)$, where $x_{1}, x_{2}$ are distinct points. For tangles $(B, t)$ and $\left(B, t^{\prime}\right)$ with $\partial t=\partial t^{\prime}$, we say that they are equivalent if there is a pairwise homeomorphism $h:(B, t) \rightarrow\left(B, t^{\prime}\right)$ satisfying $\left.h\right|_{\partial B}=$ id.

Let $U$ be the unit 3 -ball in $\mathbb{R}^{3}$, and take 4 points $\mathrm{NW}, \mathrm{NE}, \mathrm{SE}, \mathrm{SW}$ on the boundary of $U$ so that $\mathrm{NW}=(0,-\alpha, \alpha), \mathrm{NE}=(0, \alpha, \alpha), \mathrm{SE}=(0, \alpha,-\alpha)$, SW $=$ $(0,-\alpha,-\alpha)$, where $\alpha=\frac{1}{\sqrt{2}}$. A tangle $(U, t)$ is a rational tangle if it is a trivial tangle with $\partial t=\{\mathrm{NW}, \mathrm{NE}, \mathrm{SE}, \mathrm{SW}\}$. We can construct rational tangles from sequences of integers $a_{1}, a_{2}, \ldots, a_{n}$ as shown in Figure 2.1, where the last horizontal twist $a_{n}$ may be 0 . We consider that the tangle diagrams in Figure 2.1 is drawn on the $y z$-plane. Denote by $R\left(a_{1}, a_{2}, \ldots, a_{n}\right)$ the associated rational tangle.

Each rational tangle can be parametrized by $r \in \mathbb{Q} \cup\{\infty\}$, where the rational number $r$ is given by the continued fraction below. Thus we denote the rational tangle corresponding to $r$ by $R(r)$. 


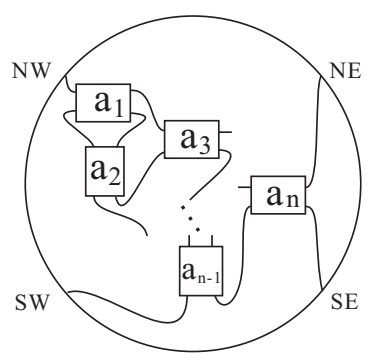

(i) $\mathrm{n}$ is odd

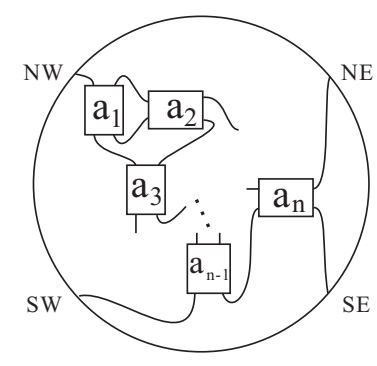

(ii) $\mathrm{n}$ is even
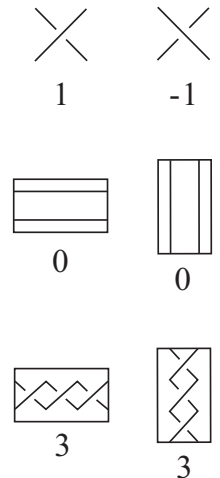

Figure 2.1. Rational tangles

$$
r=a_{n}+\frac{1}{a_{n-1}+\frac{1}{\ddots}}
$$

Let $(U, t)$ be the rational tangle $R(\infty)$. Considering $t$ is embedded in the $y z-$ plane, take the disk $D$ in the $y z$-plane such that $\partial D$ is the union of $t$ and two $\operatorname{arcs}$ in $\partial U$ : one connects NW and NE, and the other connects SW and SE. We call an arc in $D$ connecting the components of the interior of $t$ a spanning arc, and the arc $D \cap \partial U$ connecting NW and NE the latitude of $R(\infty)$. See Figure 2.2. The two-fold cover $\widetilde{U}$ of $U$ branched along $t$ is a solid torus. Note that the preimages of the spanning arc and the latitude are a core and a longitude $\lambda$ of the solid torus, respectively. A meridian of a rational tangle $R(r)=\left(U, t^{\prime}\right)$ is a simple closed curve in $\partial U-t^{\prime}$ which bounds a disk in $U-t^{\prime}$ and a disk in $\partial U$ meeting $t^{\prime}$ in two points. Let $\mu_{r}(\subset \partial \widetilde{U})$ be a lift of a meridian of $R(r)$; then $\mu_{\infty}$ is a meridian of the solid torus $\widetilde{U}$. Furthermore, we note the following well-known fact.

Lemma 2.1. Under adequate orientations we have $\left[\mu_{r}\right]=-p\left[\mu_{\infty}\right]+q[\lambda] \in H_{1}(\partial \widetilde{U})$, where $r=\frac{p}{q}$ and $\left[\mu_{\infty}\right] \cdot[\lambda]=1$.

Let $(B, t)$ be a tangle such that $B \subset S^{3}\left(=\mathbb{R}^{3} \cup\{\infty\}\right)$ is the complement of the unit 3-ball $U$, and $\partial t=\{\mathrm{NW}, \mathrm{NE}, \mathrm{SE}, \mathrm{SW}\}$. We denote by $(B, t)+R(r)$ the knot or link in $S^{3}$ formed by the union of the strings of the tangles, and let $\pi_{r}: X_{r} \rightarrow S^{3}=B \cup U$ be the two-fold cover branched along $(B, t)+R(r)$.

Suppose that $(B, t)+R(\infty)$ is a trivial knot. Then the two-fold cover $X_{\infty}$ branched along $(B, t)+R(\infty)$ is the $S^{3}$, and the preimage of the spanning arc $\kappa$ for $R(\infty)$ is a knot in $X_{\infty}=S^{3}$, which we call the covering knot of $\kappa$. The exterior of the covering knot $K$ is $\pi_{\infty}^{-1}(B)$. For $(B, t)+R(\infty)$ a replacement of $R(\infty)$ by a rational tangle $R(s)$ is called $s$-untangle surgery on $(B, t)+R(\infty)$. Performing 


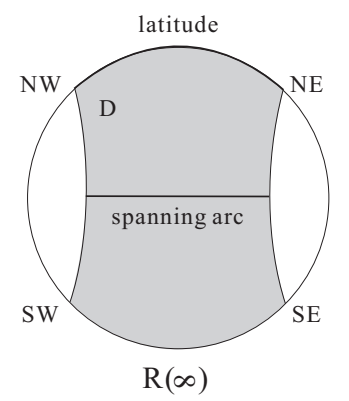

Figure 2.2. A spanning arc and a latitude

untangle surgery downstairs corresponds to replacing the solid torus $\pi_{\infty}^{-1}(U)$ by $\pi_{s}^{-1}(U)$ upstairs, i.e. Dehn surgery on the covering knot $K$. This observation is referred to as the Montesinos trick [13]. We denote the surgery slope by $\gamma_{s}$; it is represented by a lift of a meridian of $R(s)$. We say that $\gamma_{s}$ is the covering slope of $s$. See the commutative diagram below.

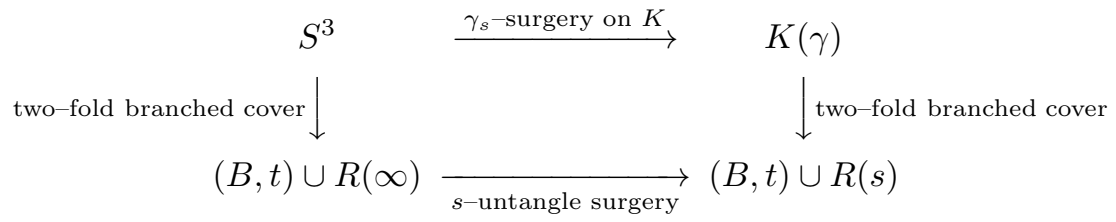

Diagram 2. Montesinos trick

For a link $L$ and an $\operatorname{arc} \kappa$ with $\kappa \cap L=\partial \kappa$ we perform an untangle surgery along $\kappa$ as follows. First take a regular neighborhood $N(\kappa)$ of $\kappa$ so that $(N(\kappa), N(\kappa) \cap L)$ is a trivial tangle. Then, identifying the trivial tangle $T=(N(\kappa), N(\kappa) \cap L)$ with the rational tangle $R(\infty)$, we can replace $R(\infty)=T$ by a rational tangle $R(s)$; this operation is called $s$-untangle surgery of $L$ along $\kappa$. Note that the definition of $s$-untangle surgery along $\kappa$ relies on the identification of $T$ with $R(\infty)$. If $L$ is a trivial knot, the two-fold cover of $S^{3}$ branched along $L$ is $S^{3}$, and the preimage of $\kappa$ is a knot, which we call the covering knot of $\kappa$. Then, as before, performing $s$-untangle surgery along $\kappa$ downstairs corresponds to performing Dehn surgery on the covering knot upstairs; we call its surgery slope the covering slope.

\section{Almost alternating unknots and L-SPaCe Knots}

A diagram of a knot is alternating if over-crossings and under-crossings alternate while running along the diagram. A diagram of a knot is almost alternating if the diagram is obtained by a single crossing change in an alternating diagram. Hence an almost alternating diagram has a crossing at which the crossing change makes the diagram alternating. Such a crossing is called a dealternator of the almost alternating diagram. For later convenience, we call an arc connecting an over pass 
and an under pass at the dealternator a dealternating arc. See Figure 3.1. (In the diagram, there are four dealternating arcs at each dealternator, but obviously they are isotopic.)

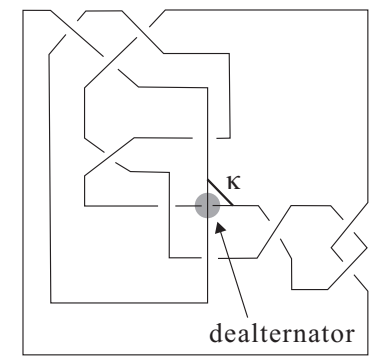

FigURE 3.1. $\kappa$ is a dealternating arc.

The following result is useful to obtain an $L$-space knot, which was observed by Ozsváth-Szabó [15, 8.3]. For completeness we give its proof here.

Theorem 3.1 (Ozsváth-Szabó). Let $O$ be a trivial knot in $S^{3}$ and $\kappa$ an arc satisfying $\kappa \cap O=\partial \kappa$. Suppose that $O$ has an almost alternating diagram with $\kappa$ a dealternating arc. Then the covering knot $K$ of $\kappa$ is an $L$-space knot.

Proof of Theorem 3.1. First isotope $O \cup \kappa$ to a position so that the diagram of $O$ is almost alternating and $\kappa$ is a dealternating arc. Consider a tangle decomposition $(B, t) \cup R(\infty)$ of the trivial knot $O$ as depicted in Figure 3.2(i). Then regard the dealternating arc $\kappa$ as a spanning arc and take the covering knot $K$ of $\kappa$. Figure 3.2 indicates that the crossing change at the dealternator (Figure 3.2(ii)) corresponds to a $(-1 / 2)$-untangle surgery of $O$ along $\kappa$ (Figure 3.2(iii)). Hence the $(-1 / 2)-$ untangle surgery along $\kappa$ converts $O$ into an alternating knot $L$ whose alternating diagram is obtained from the almost alternating diagram of $O$ by crossing change at the dealternator. Since the two-fold branched cover of $S^{3}$ branched along a non-split alternating link is an L-space [17, Lemma 3.2 and Proposition 3.3], 1/2surgery (in terms of $\left(\mu_{\infty}, \lambda\right)$-framing which may not be a preferred framing) on the covering knot $K$ yields an L-space. Thus $K$ is an L-space knot. $\square$ Theorem 3.1)

Remark 3.2. By construction, L-space knots obtained in Theorem 3.1 are strongly invertible.

Theorem 3.1 shows that each almost alternating diagram of the unknot yields an L-space knot. So it is important to find such diagrams of the unknot. For this purpose, we recall a result of Tsukamoto [22].

A reduced diagram is one not containing any nugatory crossings (Figure 3.3).

Denote by $C_{m}$ a basic almost alternating diagram of the unknot depicted in Figure 3.4(i). Then Tsukamoto [22] has shown that any reduced almost alternating diagram of the unknot can be obtained from $C_{m}$ by using only certain types of 


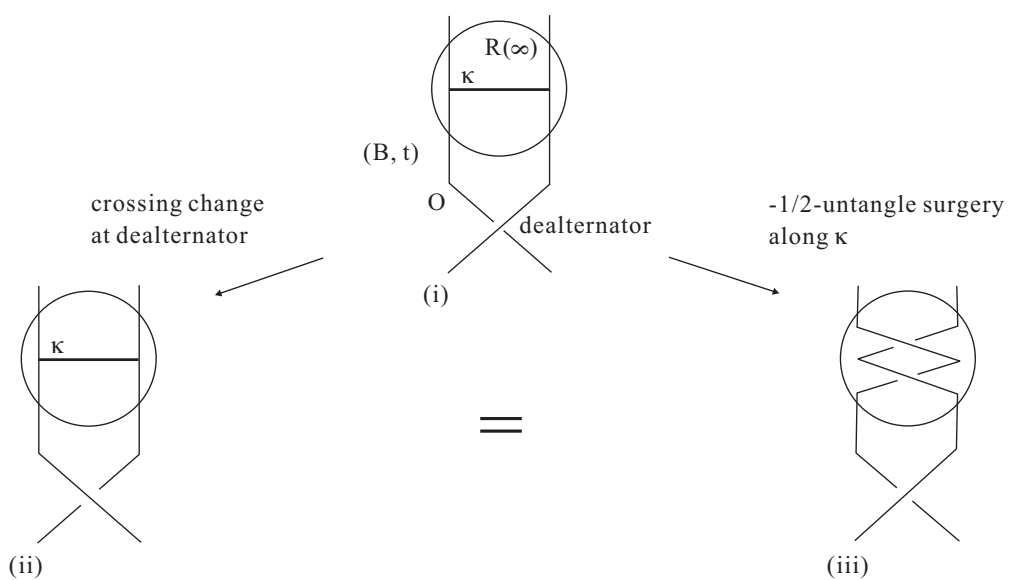

Figure 3.2. Crossing change at the dealternator and $(-1 / 2)-$ untangle surgery along the dealternating $\operatorname{arc} \kappa$

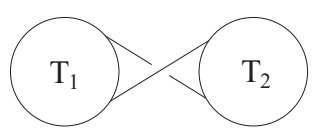

Figure 3.3. A nugatory crossing

isotopies called flypes, tongue moves and twirl moves; see Figure 3.4(ii). Recently McCoy [12] has given an alternative proof of this result.

Theorem 3.3 ([22, 12]). Any reduced almost alternating diagram of the unknot can be obtained from $C_{m}$ for some non-zero integer $m$, by a sequence of flypes, tongue moves and twirl moves.

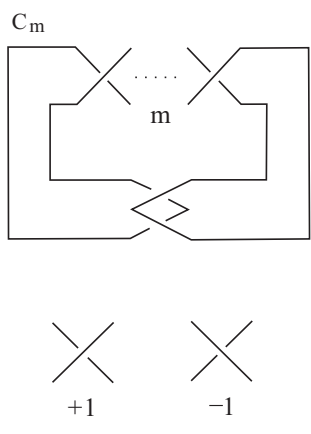

(i)
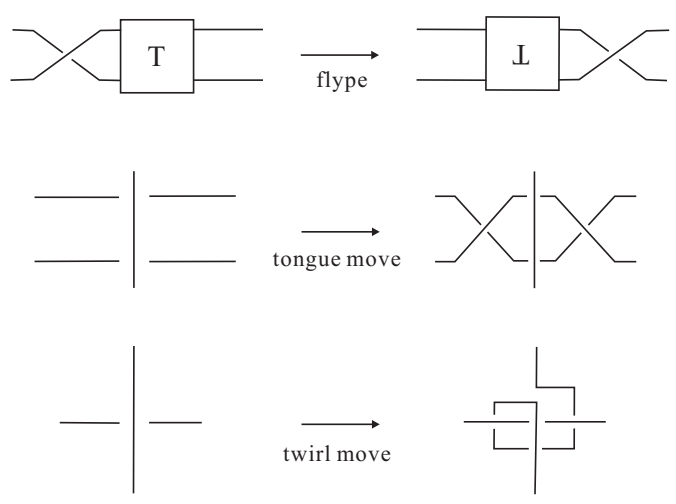

(ii)

Figure 3.4. A basic almost alternating diagram $C_{m}$ of the unknot, flype, tongue move and twirl move 


\section{A hyperbolic, L-Space KnOt With no EXCEPtional SURGERIES}

In this section we will prove Theorem 1.2 by giving a concrete example of a hyperbolic, L-space knot $K$ which has no exceptional surgeries, i.e. every nontrivial surgery on $K$ yields a hyperbolic $3-$ manifold.

Theorem 4.1. Let $K$ be a knot depicted in Figure 4.1. Then $K$ is a hyperbolic, L-space knot which has no exceptional surgeries. In particular, it admits no Seifert fibered surgeries.

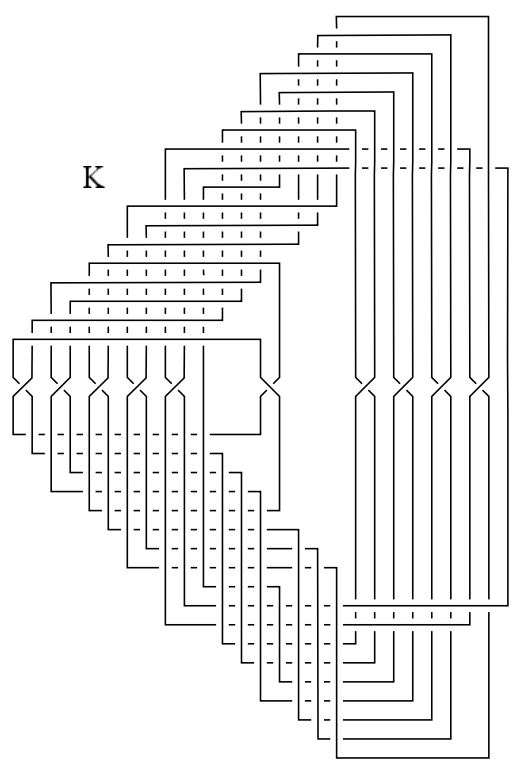

FigurE 4.1. A hyperbolic, L-space knot with no exceptional surgeries

Proof of Theorem 4.1. Based on Theorem 3.3 we will apply flypes and tongue moves to $C_{k}$ several times to obtain a sufficiently complicated almost alternating diagram of the unknot. We start with the almost alternating diagram $C_{-3}$ of the unknot and apply a sequence of flypes and tongue moves as depicted in Figures 4.2 and 4.3 to obtain the almost alternating diagram of the unknot given by the last picture of Figure 4.3.

Let $\kappa$ be a dealternating arc as in Figure 4.4. To obtain an explicit picture of the covering knot $K$ of $\kappa$, we apply isotopies given in Figures 4.4-4.9. Then taking the two-fold cover branched along $O$, we obtain the covering knot $K$ of $\kappa$; see Figure 4.10. By Theorem $3.1 K$ is an L-space knot.

A proof showing that $K$ is a hyperbolic knot with no exceptional surgeries is computer-assisted but rigorous. SnapPy [5] finds an approximated hyperbolic structure of $K$, which can be verified by the program HIKMOT [7]. To see that $K$ has no exceptional surgeries we run fef . py written by Ichihara and Masai [9], which is 

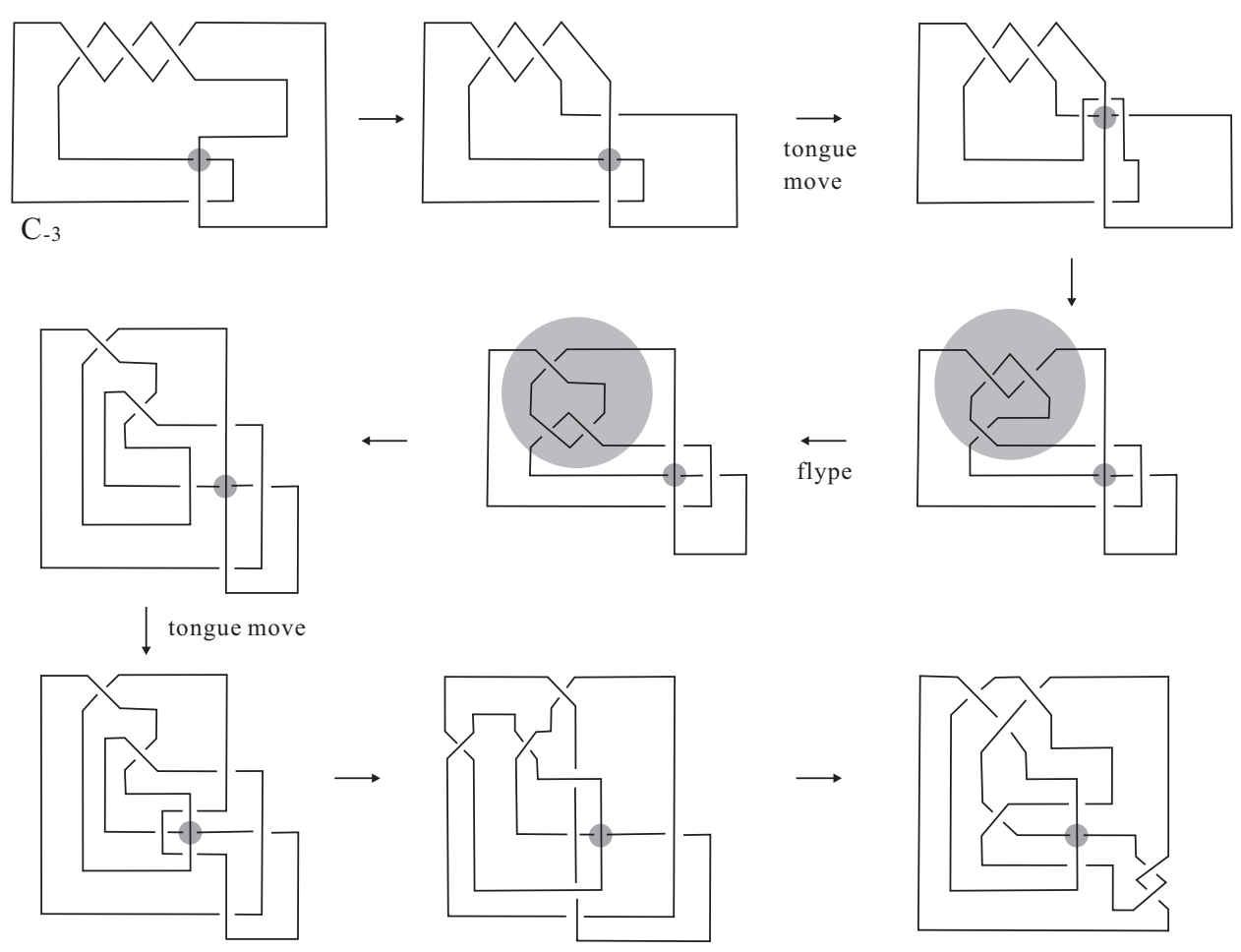

Figure 4.2. Sequence of flypes and tongue moves
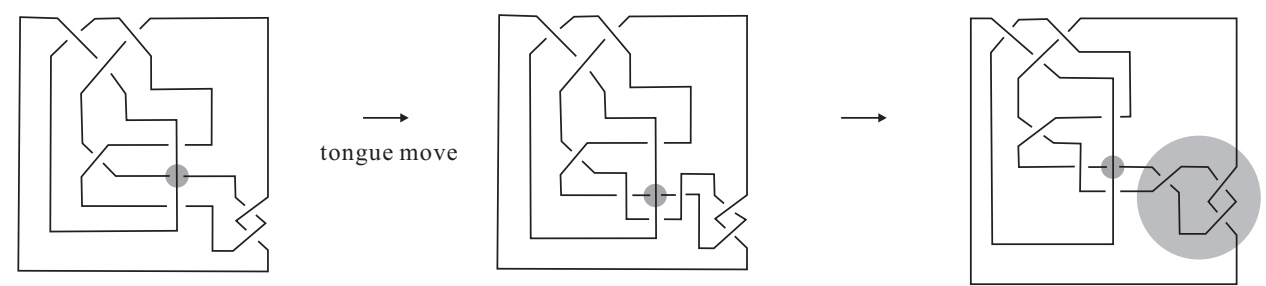

$$
\text { flype } \downarrow
$$
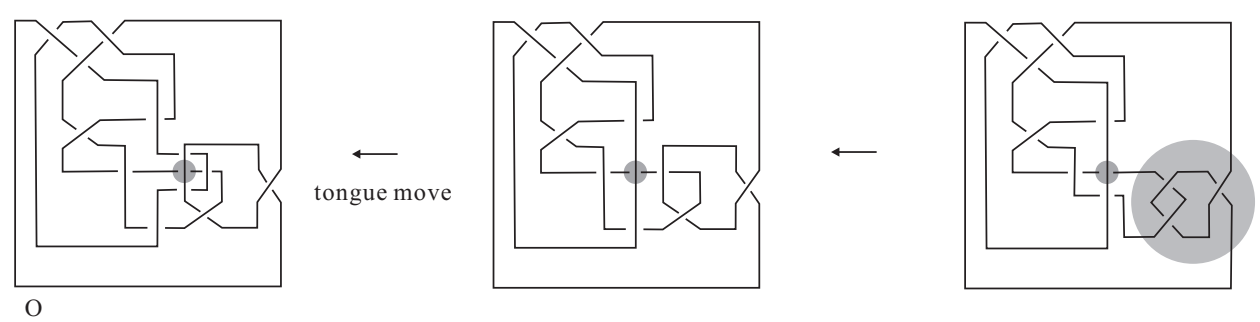

Figure 4.3. Sequence of flypes and tongue moves; continued from Figure 4.2 
a modification of a python code find_exceptional_fillings .py developed in [11]. They improved the codes in [11] using verified numerical analysis based on interval arithmetics to obtain mathematically rigorous computations. The code fef.py, together with HIKMOT, gives us as an output a list of candidates for exceptional fillings of $E(K)$, including all truly exceptional ones. See [9, Section 6] for detailed explanation on fef.py. For the knot $K$, the set of candidate exceptional fillings turns out to be empty, and this proves that $K$ has no exceptional surgeries.
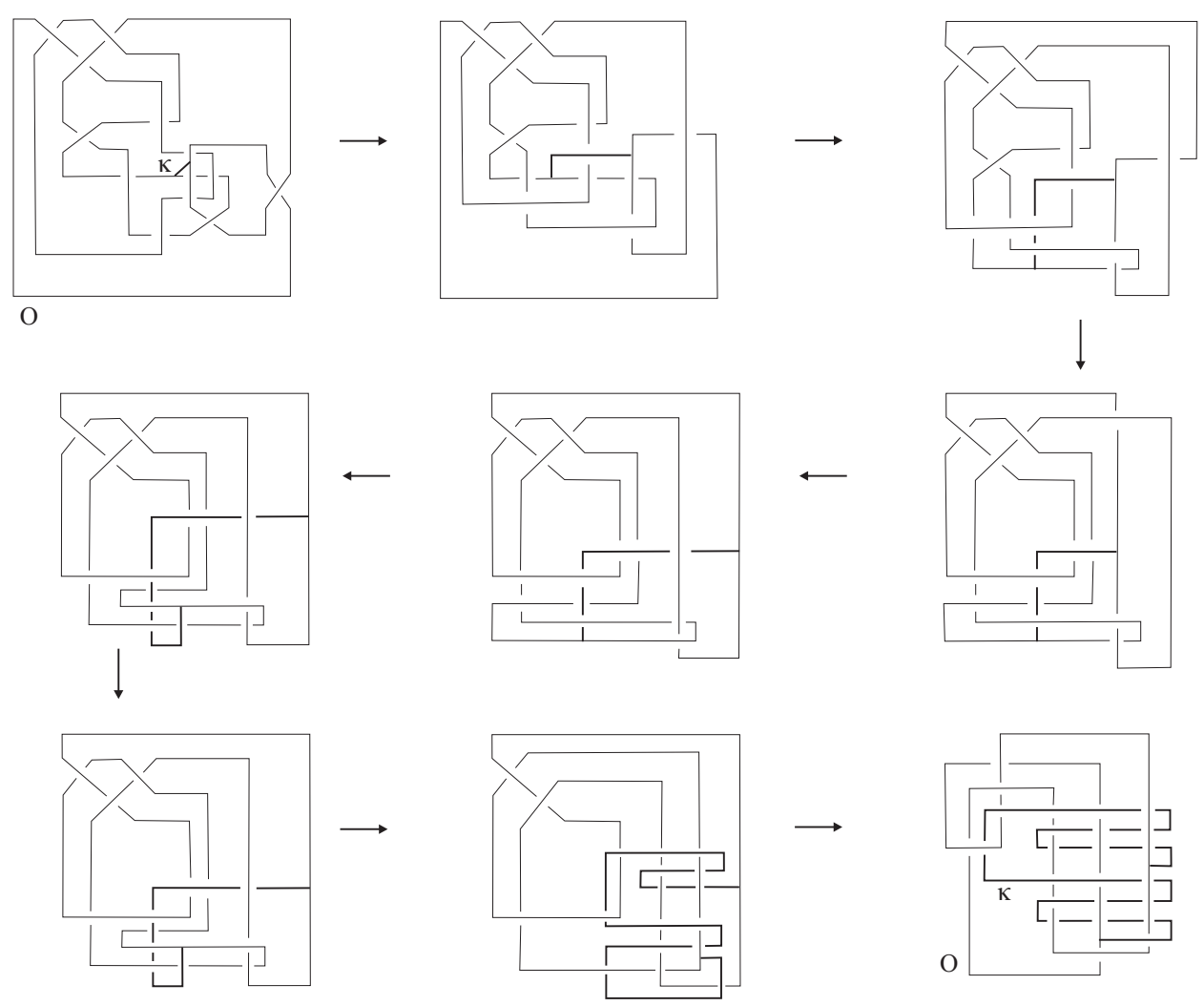

FiguRE 4.4. Isotopies of $O \cup \kappa$

Remark 4.2. In the fifth diagram in Figure 4.2 if we perform (-1)-untangle surgery along the dealternating arc $\kappa_{1}$ given in Figure 4.11(i), we obtain a twobridge knot. This implies that a surgery on the covering knot $K_{1}$ of $\kappa_{1}$ along the covering slope yields a lens space. Similarly, in the first diagram in Figure 4.3, performing 0-untangle surgery along the dealternating arc $\kappa_{2}$ given in Figure 4.11(ii) yields also a two-bridge knot. Thus the covering knot $K_{2}$ of $\kappa_{2}$ has a lens space surgery. 


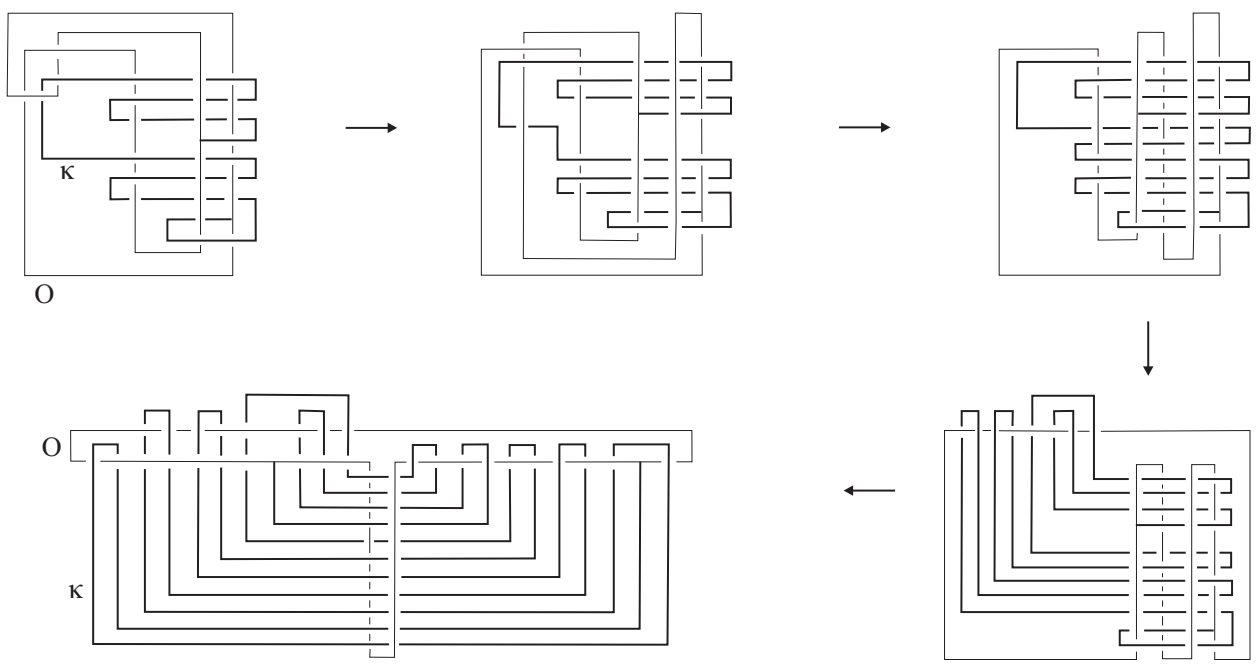

FiguRE 4.5. Isotopies of $O \cup \kappa$; continued from Figure 4.4
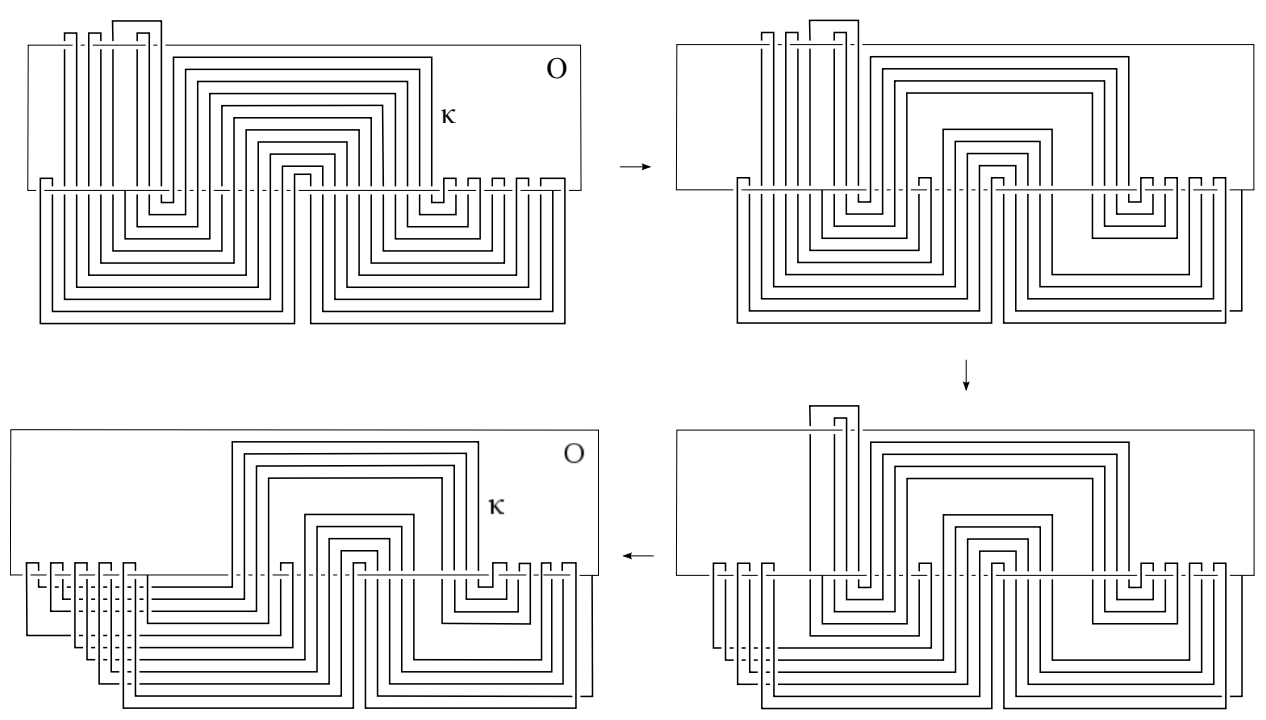

Figure 4.6. Isotopies of $O \cup \kappa$; continued from Figure 4.5

\section{REFERENCES}

[1] K. Baker and A. Moore; Montesinos knots, Hopf plumbings, and L-space surgeries, preprint 2014.

[2] R. Benedetti and C. Petronio; Lectures on hyperbolic geometry, Universitext, SpringerVerlag, 1992.

[3] J. Berge; Some knots with surgeries yielding lens spaces, unpublished manuscript.

[4] M. Boileau and J. Porti; Geometrization of 3-orbifolds of cyclic type, Astérisque 272 (2001), 208pp.

[5] M. Culler, N. Dunfield, and J. R. Weeks; SnapPy, a computer program for studying the geometry and topology of 3-manifolds, http://snappy.computop.org. 

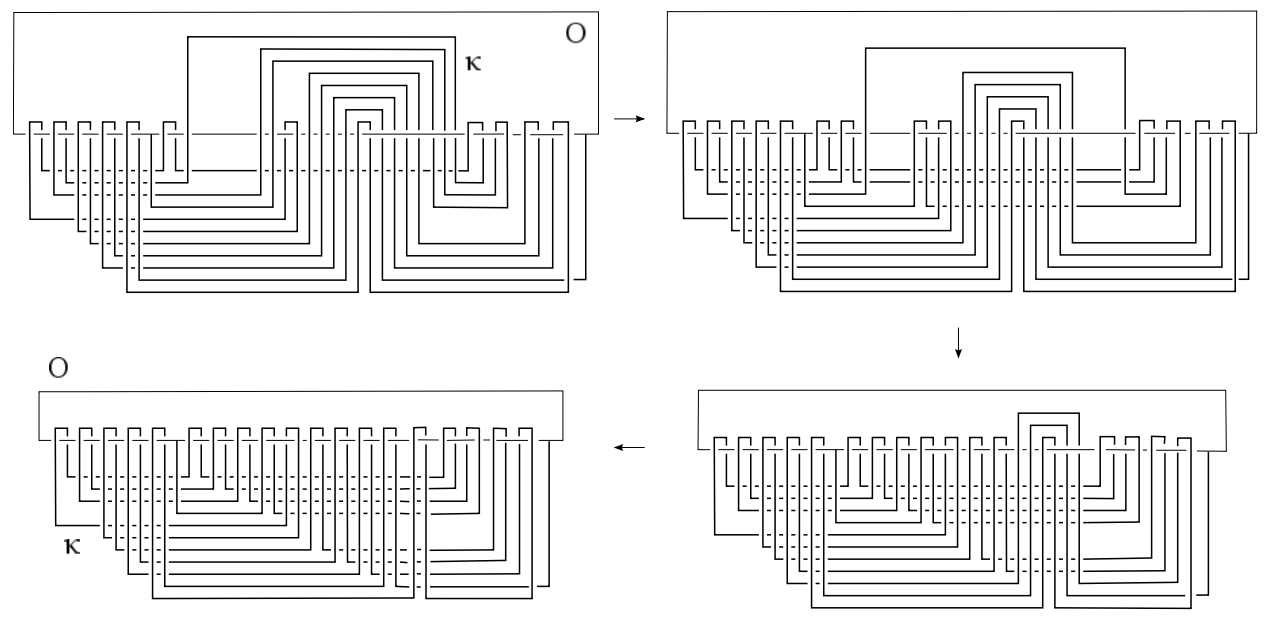

Figure 4.7. Isotopies of $O \cup \kappa$; continued from Figure 4.6

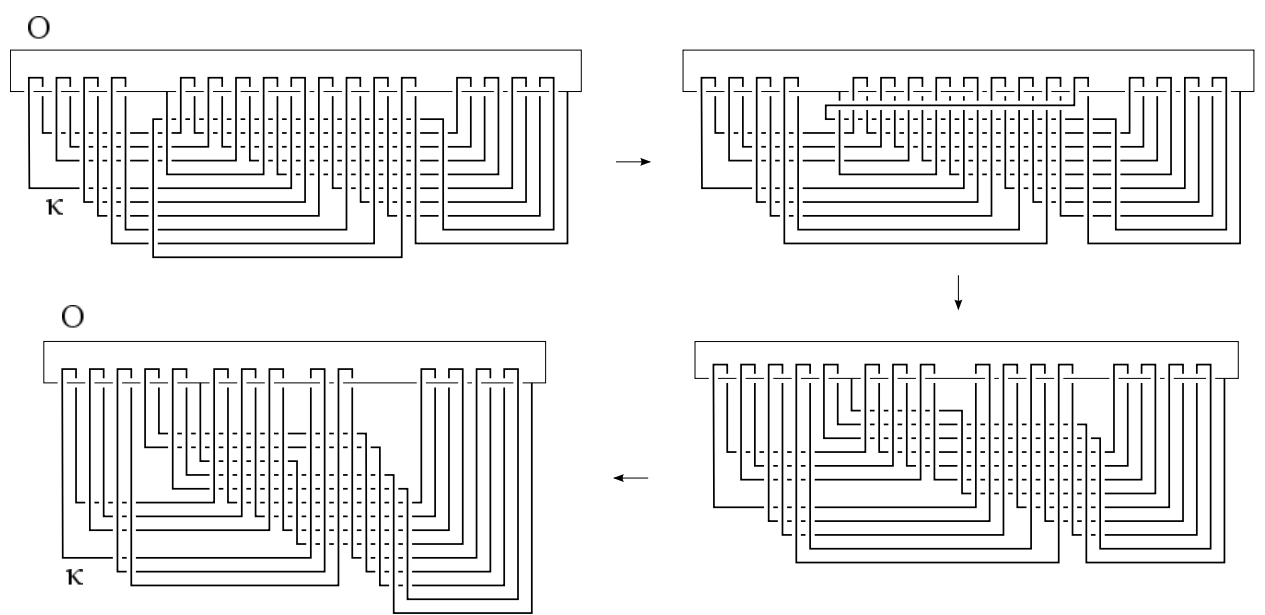

FIGURE 4.8. Isotopies of $O \cup \kappa$; continued from Figure 4.7

[6] M. Hedden; On knot Floer homology and cabling II, Int. Math. Res. Not. IMRN, (12):2248$2274,2009$.

[7] N. Hoffman, K. Ichihara, M. Kashiwagi, H. Masai, S. Oishi, and A. Takayasu; Verified computations for hyperbolic 3-manifolds, preprint 2013.

[8] J. Hom, T. Lidman and F. Vafaee; Berge-Gabai knots and L-space satellite operations, preprint 2014

[9] K. Ichihara and H. Masai; Exceptional surgeries on alternating knots, preprint 2014.

[10] T. Lidman and A. Moore; Pretzel knots with L-space surgeries, preprint 2013.

[11] B. Martelli, C. Petronio, and F. Roukema; Exceptional Dehn surgery on the minimally twisted five-chain link, Comm. Anal. Geom. 22 (2014) 689-735.

[12] D. McCoy; Alternating knots with unknotting number one, preprint 2014.

[13] J. M. Montesinos; Surgery on links and double branched coverings of $S^{3}$, Knots, groups, and 3-manifolds (Papers dedicated to the memory of R.H.Fox), Ann. Math. Studies, 84, Princeton Univ. Press 1975, 227-259, 

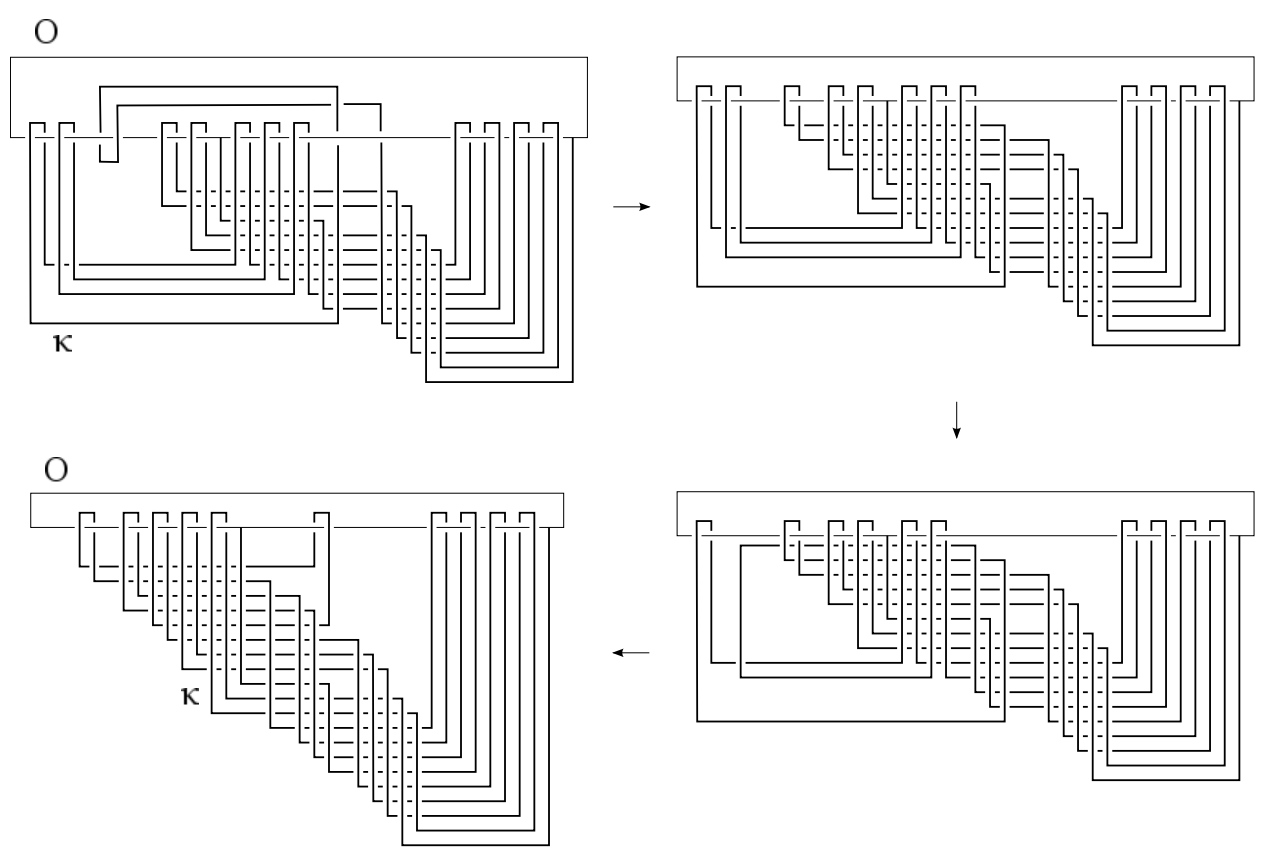

FIGURE 4.9. Isotopies of $O \cup \kappa$; continued from Figure 4.8

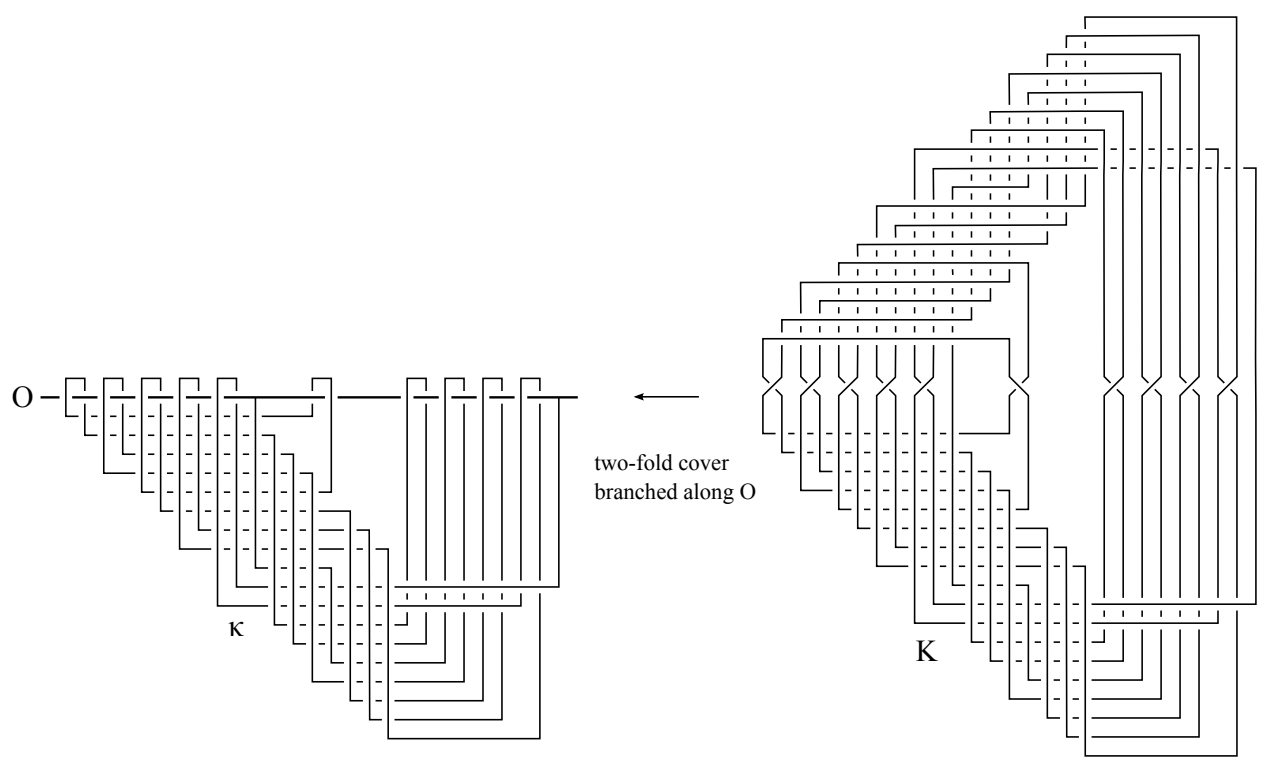

Figure 4.10. The covering knot $K$ of $\kappa$

[14] K. Motegi; L-space surgery and twisting operation, preprint 2014.

[15] P. Ozsváth and Z. Szabó; Knots with unknotting number one and Heegaard Floer homology, Topology 44 (2005), 705-745. 


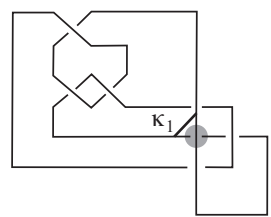

(i)
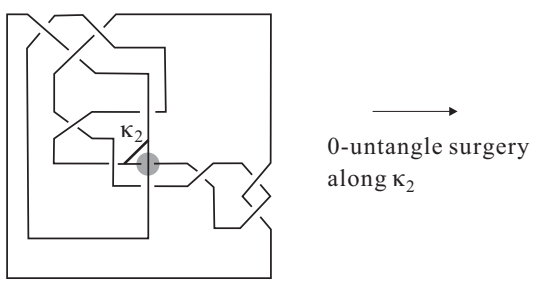
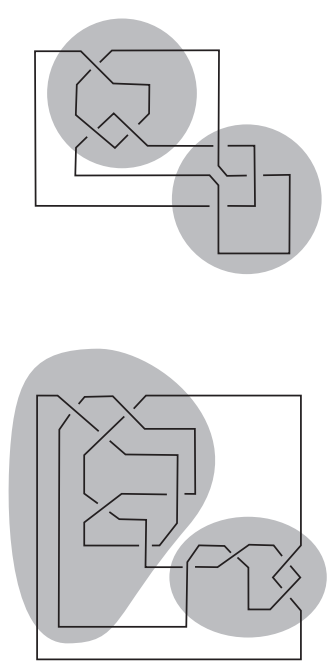

(ii)

Figure 4.11. ( -1$)$-untangle surgery along $\kappa_{1}$ yields a two-bridge knot, and $0-$-untangle surgery along $\kappa_{2}$ yields a two-bridge knot.

[16] P. Ozsváth and Z. Szabó; On knot Floer homology and lens space surgeries, Topology 44 (2005), 1281-1300.

[17] P. Ozsváth and Z. Szabó; On the Heegaard Floer homology of branched double-covers. Adv. Math. 194 (2005), 1-33.

[18] P. Ozsváth and Z. Szabó; Knot Floer homology and rational surgeries, Algebr. Geom. Topol. 11 (2011), 1-68.

[19] C. Petronio and J. Porti; Negatively oriented ideal triangulations and a proof of Thurston's hyperbolic Dehn filling theorem, Expo. Math. 18 (2000), 1-35.

[20] W. P. Thurston; The geometry and topology of 3-manifolds, Lecture notes, Princeton University, 1979.

[21] W. P. Thurston; Three dimensional manifolds, Kleinian groups and hyperbolic geometry, Bull. Amer. Math. Soc. 6 (1982), 357-381.

[22] T. Tsukamoto; The almost alternating diagram of the trivial knot, J. Topology 2 (2009), $77-104$.

Department of Mathematics, Nihon University, 3-25-40 Sakurajosui, Setagaya-ku, TOKYO 156-8550, JAPAN

E-mail address: motegi@math.chs.nihon-u.ac.jp

Graduate School of Integrated Basic Sciences, Nihon University, 3-25-40 SakurajoSui, Setagaya-ku, Tokyo 156-8550, Japan 